\title{
Improving Decannulation and Swallowing Function: A Comprehensive, Multidisciplinary Approach to Post-Tracheostomy Care
}

\author{
John W Mah MD, Ilene I Staff PhD, Sylvia R Fisher SLP, and Karyn L Butler MD
}

BACKGROUND: Multidisciplinary tracheostomy teams have been successful in improving operative outcomes; however, limited data exist on their effect on postoperative care. We aimed to determine the effectiveness of a multidisciplinary tracheostomy service alone and following implementation of a post-tracheostomy care bundle on rates of decannulation and tolerance of oral diet before discharge. METHODS: Prospective data on all subjects requiring tracheostomy by any trauma/ critical care surgeon were collected from January 2011 to December 2013 following development of a tracheostomy service and continued following implementation of the post-tracheostomy care bundle. Rates of decannulation and tolerance of oral diet were compared between all groups: pre-tracheostomy service (baseline, historical control), tracheostomy service alone, and tracheostomy service with post-tracheostomy care bundle. RESULTS: Three hundred ninety-three subjects met the criteria for analysis with 61 in the baseline group, 124 following initiation of a tracheostomy service, and 208 after the addition of the post-tracheostomy care bundle. There were significant overall differences between all groups in the proportion of subjects decannulated, proportion of subjects tolerating oral diet, and number of subjects receiving speech evaluations. Pairwise comparisons showed no differences in decannulation or tolerance of oral diet following implementation of the tracheostomy service alone but significant improvement with the addition of the posttracheostomy care bundle compared with baseline. $(P=.002$ and $P=.005$, respectively). Likewise, the number of speech language pathologist consults significantly increased compared with baseline only after the post-tracheostomy care bundle $(P=.004)$. Time to speech evaluation significantly decreased with the post-tracheostomy care bundle compared with baseline and tracheostomy service $(P<.013)$. CONCLUSIONS: The addition of a post-tracheostomy care bundle to a multidisciplinary tracheostomy service significantly improved rates of decannulation and tolerance of oral diet. Key words: tracheostomy; tracheotomy; respiratory failure; patient care team; quality of life; postoperative care; speech therapy. [Respir Care 2017;62(2):137-143. (C) 2017 Daedalus Enterprises]

\section{Introduction}

Placement of a tracheostomy tube may be necessary for patients in the ICU with respiratory failure. In fact, the

\footnotetext{
Drs Mah and Butler are affiliated with Critical Care, Department of Surgery, Hartford Hospital, University of Connecticut School of Medicine, Hartford, Connecticut. Dr Staff is affiliated with the Department of Research, Hartford Hospital, Hartford, Connecticut. Ms Fisher is affiliated with Speech Therapy, Department of Rehabilitation Services, Hartford Hospital, Hartford, Connecticut.
}

Dr Mah has disclosed a relationship with Smiths-Medical. The other authors have disclosed no conflicts of interest.

Dr Mah presented a version of this work at the Society of Critical Care Medicine Conference, held January 19-23, 2013, in San Jose, Puerto Rico. incidence of tracheostomy seems to be increasing out of proportion to the increased need for mechanical ventilation. ${ }^{1}$ This has led some hospitals to develop tracheostomy teams to standardize and deliver specialized patient care to reduce perioperative tracheostomy-related complications. $^{2-6}$ However, postoperative tracheostomy care outside the ICU setting is often overshadowed by the primary disease processes, and tracheostomy care is typically

\footnotetext{
Correspondence: John W Mah MD, Department of Surgery, 80 Seymour Street, Hartford Hospital, Hartford, CT 06102. E-mail: John.Mah@hhchealth.org.
}

DOI: $10.4187 /$ respcare. 04878 
delivered by multiple providers, including the primary physician, resident, mid-level providers, consulting surgeon, nurse, respiratory therapist, and speech language pathologist, each relying on the others to act independently. Thus, many patients may receive fragmented and suboptimal postoperative tracheostomy care, often neglecting a thorough evaluation of their individual tracheostomy needs, including advancement of diet or ongoing need for tracheostomy. ${ }^{7}$

\section{See the Related Editorial on Page 246}

In fact, the presence of a tracheostomy tube may impair swallowing function. ${ }^{8-15}$ Farri et $\mathrm{al}^{16}$ found that in subjects with tracheostomy, following head and neck surgery, dysphagia negatively impacted emotional and social outcomes. More importantly, quality of life in these subjects was improved by providing education from a healthcare professional on how to improve their swallowing function. ${ }^{16}$ Similarly, in non-cancer patients with tracheostomy, decannulation improved quality of life as a result of enhanced body image perception. ${ }^{17}$ Although more substantial data are limited, it seems rational to believe that advancing patients to a state of tolerating oral nutrition and eliminating reliance on artificial devices would improve a patient's quality of life. Unfortunately, few studies have examined this progression in regard to more frequent decannulations, improvements in speech function, or tolerance of an oral diet, with many studies limited by design, power, or validity. ${ }^{18}$

With the success of bundled therapy ${ }^{19}$ in multiple other areas of medicine, including reduction in urinary tract infections, sepsis, ventilator-associated pneumonia, and catheter-related bloodstream infections, ${ }^{20-23}$ we hypothesized that adding a post-tracheostomy care bundle to our existing tracheostomy service would improve rates of decannulation and swallowing function. The bundle included a process to identify and follow all patients immediately after tracheostomy, established a consistent method to deliver postoperative care, and organized all essential providers with the goal of progressing all patients to their maximal clinical potential.

\section{Methods}

\section{Study Population}

This is a prospective cohort study designed to examine the impact of a comprehensive postoperative care bundle on rates of decannulation and swallowing function. Subjects undergoing tracheostomy before implementation of the bundle served as historical controls. The study was approved by the institutional review board of Hartford

\section{QUICK LOOK}

\section{Current knowledge}

Tracheostomy teams have focused on improving perioperative outcomes. Only a few studies focusing on care outside the ICU demonstrated an improvement in time to decannulation, with none showing improvement in overall rates of decannulation. There is also little to no evidence of assessment and/or improvement in diet or swallowing function following tracheostomy.

\section{What this paper contributes to our knowledge}

This paper draws attention to the need for better posttracheostomy care. Tracheostomy teams alone were not able to improve rates of decannulation or tolerance of oral diet. Via the addition of a simple post-tracheostomy care bundle, the intensivist-led tracheostomy team was able to make a significant improvement in the care of subjects following tracheostomy.

Hospital (Hartford, Connecticut). Hartford Hospital is an 800-bed tertiary referral, level 1 trauma center with $>22,000$ ICU admissions during the study period. Data collected on all subjects from March 2010 to December 2013 , over the age of 18 , who underwent tracheostomy by a trauma/critical surgeon, were stored in the Surgical Critical Care Tracheostomy Database and eligible for the study. Patients were excluded if they did not survive their hospital stay or were in a withdrawal of support process. Patients undergoing tracheostomy by any ear, nose, and throat surgeon were not included.

Three groups were categorized according to three specific implementation strategies and corresponding consecutive time periods. A retrospective control group was identified and included all subjects receiving tracheostomy from March 2010 to December 2010 before development of a formal tracheostomy service (baseline group). Prospective data were then collected marking the implementation of an intensivist-led tracheostomy service, which included all subjects receiving a tracheostomy from January 2011 to December 2011 (tracheostomy service group). Finally, a post-tracheostomy care bundle was introduced, and prospective data were collected on all subjects receiving tracheostomy from January 2012 to December 2013, encompassing the intensivist-led tracheostomy service and implementation of the post-tracheostomy care bundle (posttracheostomy care bundle group).

\section{Post-Tracheostomy Care Bundle}

The post-tracheostomy care bundle consisted of 4 parts: an electronic postoperative order set with automated re- 
Table 1. Post-Tracheostomy Care Bundle

\begin{tabular}{|c|c|}
\hline Bundle & Description \\
\hline $\begin{array}{l}\text { Electronic postoperative tracheostomy } \\
\text { order set }\end{array}$ & $\begin{array}{l}\text { Standardized preselected order set that contains: } \\
\text { (1) Automatic RT consult } \\
\text { (2) Immediate SLP consult } \\
\text { (3) Detailed description of }(\mathrm{RN}) \text { tracheostomy care } \\
\text { (4) Common problem list and instructions } \\
\text { (5) Specific emergency instructions } \\
\text { (6) Team contact information }\end{array}$ \\
\hline Tracking and following system & $\begin{array}{l}\text { All patients with tracheostomy or receiving tracheostomy care require electronic documentation and } \\
\text { orders for respiratory therapy. The electronic patient medical record was accessed to identify all } \\
\text { patients with tracheostomy, including patients who failed to have the post-tracheostomy order set or } \\
\text { new patients being admitted with a pre-existing tracheostomy. }\end{array}$ \\
\hline Decannulation protocol & $\begin{array}{l}\text { SLP collaborates with RT and surgeon and bedside RN to begin speaking valve trials and assess for } \\
\text { downsizing to improve swallowing function. RT is empowered to evaluate, change/downsize, and } \\
\text { decannulate with guidance from the covering tracheostomy surgeon. The surgeon is required to change } \\
\text { the tracheostomy tube if there is an immature tract. }\end{array}$ \\
\hline Tracheostomy rounds & $\begin{array}{l}\text { Weekly rounds consisted of a tracheostomy surgeon, RT, and SLP. This process utilized the tracking and } \\
\text { following system to generate an accurate list of all patients with a tracheostomy. } \\
\text { The rounding process: } \\
\text { (1) Established the appropriate plan of care } \\
\text { (2) Identified patients who could have their tracheostomy tubes downsized or decannulated } \\
\text { (3) Identified patients who could have diets started or advanced } \\
\text { (4) Provided education and awareness to staff regarding tracheostomy care }\end{array}$ \\
\hline $\begin{array}{l}\mathrm{RT}=\text { respiratory therapist } \\
\mathrm{SLP}=\text { speech language pathologist } \\
\mathrm{RN}=\text { registered nurse }\end{array}$ & \\
\hline
\end{tabular}

spiratory therapist and speech language pathologist consultations, a tracheostomy tracking and following system, a respiratory therapist-driven decannulation protocol, and a formal rounding process to individualize tracheostomy care (Table 1).

\section{Data Collection}

The primary outcome was tracheostomy decannulation before hospital discharge. Secondary outcomes included tolerance of oral diet and speech consultation by day 2 . Diet tolerance was determined by a yes-no assessment with "yes" being defined as tolerating a National Dysphagia Diet 1 or better and "no" to all other subjects. Both initial and best diets were recorded. The time to speech consultation by day 2 was collected as a dichotomous variable. Additional subject demographics, tracheostomy characteristics, and stay were collected (Table 2).

\section{Data Analysis}

Decannulation and occurrence of speech evaluation were analyzed with independent group chi-square tests of proportion. Preliminary analysis compared the subjects in each of the 3 cohorts for demographics, severity of illness, and tracheostomy characteristics using chi-square tests for di- chotomous or categorical variables and either one-way analysis of variance or the Kruskal-Wallis test for continuous variables. Kruskal-Wallis and chi-square tests of proportion were used for the continuous and dichotomous measures of time until speech evaluation. In addition to the analyses indicating an overall difference among the 3 groups, 3 additional analyses were run using chi-square tests, $t$ tests, or Wilcoxon ranked sum tests for pairwise comparison. Given the multiple testing, a Bonferroni correction was applied, and $P<.0125$ was considered significant for these analyses.

A multivariate logistic regression predicting decannulation was performed using time period/implementation strategy as the key predictor of interest and any other factors showing a univariate relationship with time period or decannulation as covariates. Data were compiled in Excel and transferred to SPSS 21 (IBM, Armonk, New York) for analysis.

\section{Results}

\section{Characteristics of the 3 Study Groups}

From March 2010 to December 2013, 477 patients met the criteria, with 84 patients excluded due to death or withdrawal of support, leaving 393 subjects available for 
Table 2. Subject Characteristics

\begin{tabular}{|c|c|c|c|c|}
\hline Characteristic & $\begin{array}{c}\text { Pre-Tracheostomy } \\
\text { Service Baseline }(n=61)\end{array}$ & $\begin{array}{l}\text { Tracheostomy Service } \\
\text { Alone }(n=124)\end{array}$ & $\begin{array}{l}\text { PTC bundle } \\
(n=208)\end{array}$ & $P$ \\
\hline Male sex, $n(\%)$ & $37(63.8)$ & $75(63.6)$ & $137(67.2)$ & .77 \\
\hline Age, mean $\pm \mathrm{SD}$ y & $56.2 \pm 16.3$ & $53.1 \pm 18.4$ & $56.2 \pm 16.7$ & .26 \\
\hline $\mathrm{BMI}$, mean $\pm \mathrm{SD} \mathrm{kg} / \mathrm{m}^{2}$ & $29.0 \pm 9.3$ & $29.6 \pm 9.0$ & $28.9 \pm 6.9$ & .73 \\
\hline Percutaneous trach, $n(\%)$ & $28(45.9)$ & $56(46.2)$ & $77(37)$ & .24 \\
\hline Prior trach, $n(\%)$ & $1(1.7)$ & $7(7.5)$ & $6(4.1)$ & .22 \\
\hline \multicolumn{5}{|l|}{ Consulting service, $n(\%)$} \\
\hline General surgery & $9(14.8)$ & $11(8.9)$ & $19(9.1)$ & .42 \\
\hline Trauma & $12(19.7)$ & $42(33.9)$ & $74(35.6)$ & \\
\hline Neurological & $25(41.0)$ & $50(40.3)$ & $72(34.6)$ & \\
\hline Cardiothoracic/vascular & $6(9.8)$ & $8(6.5)$ & $20(9.6)$ & \\
\hline General medical & $9(14.8)$ & $13(10.5)$ & $23(11.1)$ & \\
\hline SOFA, median (IQR)* & $9(7,10)$ & $6(5,7)$ & $7(6,9)$ & $<.001$ \\
\hline APACHE, median (IQR)* & $27(23.25-29)$ & $20(17-24)$ & $23(20-28)$ & $<.001$ \\
\hline Hospital LOS, median (IQR)d & $38.0(29.5-63.0)$ & $36.0(28.0)$ & $35.5(28.0-48.0)$ & .35 \\
\hline ICU LOS, median (IQR)d & $26.5(19.3-38.0)$ & $26.0(18.7-37.0)$ & $25.0(18.8-32.0)$ & .15 \\
\hline Duration of ventilation, median (IQR) $\dagger d$ & $26.0(17.0-35.0)$ & $21.5(14.3-30.8)$ & $20.0(14.0-26.0)$ & .003 \\
\hline \multicolumn{5}{|c|}{$\begin{array}{l}\text { * All three pairwise comparisons significant. } \\
\dagger \text { Baseline and post-tracheostomy bundle differ significantly. } \\
\text { PTC = post-tracheostomy care } \\
\text { BMI = body mass index } \\
\text { trach = tracheostomy } \\
\text { SOFA = Sequential Organ Failure Assessment } \\
\text { IQR = interquartile range } \\
\text { APACHE = Acute Physiology and Chronic Health Evaluation } \\
\text { LOS = length of stay }\end{array}$} \\
\hline
\end{tabular}

analysis. Sixty-one were included in the baseline data set, 124 during the period after a tracheostomy service was instituted, and 208 following implementation of the posttracheostomy care bundle.

All group characteristics were not significantly different with the exception of severity of illness and ventilator stay (Table 2). The pre-tracheostomy (baseline) service group contained the most severely ill subjects with the highest Sequential Organ Failure Assessment (SOFA) and Acute Physiology and Chronic Health Evaluation (APACHE) II scores followed by the lowest SOFA and APACHE II scores during the tracheostomy service period and significantly higher scores again during the time period following the post-tracheostomy care bundle. Duration of mechanical ventilation was significantly shorter in the posttracheostomy care bundle compared with baseline but not significantly different from the tracheostomy service group.

\section{Impact of the Tracheostomy Service and Post- Tracheostomy Care Bundle}

There were significant overall differences among the 3 study groups for proportions of subjects decannulated before discharge, tolerance of initial oral diet, and tolerance of any diet following tracheostomy. There were also significant overall differences among the 3 study groups for the proportion of subjects receiving a speech language pathologist consult and the time to receiving a speech language pathologist consult if it occurred. Pairwise comparisons between all groups show that for the main outcomes, decannulation and tolerance of oral diet, the tracheostomy service showed no statistical difference; however, with the addition of the post-tracheostomy care bundle, decannulation and tolerance of oral diet significantly improved from baseline. The post-tracheostomy care bundle also significantly improved time to speech evaluation overall and the proportion of subjects receiving a speech evaluation within $2 \mathrm{~d}$ in comparison with both the tracheostomy service alone and baseline. These comparisons are detailed in Table 3.

Logistical regression (Table 4) analysis confirm the results of the pairwise comparisons in regard to decannulation, showing that only the combination of the tracheostomy service with the post-tracheostomy care bundle was a significant predictor of successful decannulation compared with baseline $(P=.003)$. Interestingly, sex was also predictive of successful decannulation. Significantly more males achieved decannulation before discharge compared with females (24\% vs $9 \%$, $P<.001)$. APACHE II and SOFA scores had no effect as markers of severity of illness or as severity of illness indicators. 
Table 3. Comparison of Subject Outcomes

\begin{tabular}{|c|c|c|c|c|}
\hline Characteristic & $\begin{array}{l}\text { Pre-Tracheostomy Service } \\
\text { Baseline }(n=61)\end{array}$ & $\begin{array}{l}\text { Tracheostomy Service } \\
\text { Begins }(n=124)\end{array}$ & $\begin{array}{l}\text { PTC Bundle } \\
(n=208)\end{array}$ & $\begin{array}{c}P \dagger \\
\text { (Overall) }\end{array}$ \\
\hline Decannulated, $n(\%)$ & $5(8.2)$ & $18(14.5)$ & $54(26.0)^{*}$ & .002 \\
\hline Tolerate oral diet (first post-tracheostomy diet), $n(\%)$ & $10(16.4)$ & $29(23.4)$ & $72(34.6)^{*}$ & .007 \\
\hline Tolerate oral diet before leaving hospital, $n(\%)$ & $15(24.6)$ & $44(35.5)$ & $96(46.4)^{*}$ & .005 \\
\hline Receive SLP consult post-tracheostomy, $n(\%)$ & $47(77.0)$ & $109(87.9)$ & $192(92.3)^{*}$ & .004 \\
\hline $\begin{array}{l}\text { Time to speech evaluation, } \mathrm{d} \text { (among those } \\
\text { receiving), median (IQR) }\end{array}$ & $6.0(2.0-12.0)$ & $6.0(3.0-12.0)$ & $2.0(0.0-6.0)^{* *}$ & $<.001$ \\
\hline $\begin{array}{l}\text { Time to speech evaluation (among those receiving), } \\
n(\%) \text { receiving within } 2 \mathrm{~d}\end{array}$ & $12(25.2)$ & $24(22.0)$ & $108(56.3)^{* *}$ & $<.001$ \\
\hline $\begin{array}{l}\text { 3-way Kruskal-Wallis test. } \\
\text { * Significant from baseline }(P<.0125) \text {. } \\
\text { ** Significant from baseline and tracheostomy service }(P<.0125) . \\
\text { PTC = post-tracheostomy care } \\
\text { SLP = speech language pathologist } \\
\text { IQR = Interquartile range }\end{array}$ & & & & \\
\hline
\end{tabular}

Table 4. Predicting Decannulization

\begin{tabular}{lccc}
\hline \hline \multicolumn{1}{c}{ Predictor } & $\beta$ Weight & Odds Ratio & $95 \%$ CI \\
\hline Time period & & & .02 \\
Trach service vs baseline & 0.593 & 1.81 & $0.60-5.49$ \\
Trach service plus PTC bundle vs trach service & 0.637 & 1.89 & $0.98-3.63$ \\
Trach service plus PTC bundle vs baseline & 1.229 & 3.42 & $1.26-9.27$ \\
Sex (comparing males with females) & 1.207 & 3.34 & $1.70-6.58$ \\
SOFA $>6$ & -.121 & 0.886 & $0.48-1.636$ \\
APACHE $>$ 19 & 0.725 & 2.06 & .056 \\
Ventilated time (transformed to $\log _{10}$ ) & -.644 & 0.283 & $0.16-1.70$ \\
& & & .02 \\
Trach $=$ tracheostomy & & & .05 \\
PTC $=$ post-tracheostomy care & & & .53 \\
SOFA = Sequential Organ Failure Assessment & & & \\
APACHE $=$ Acute Physiology and Chronic Health Evaluation & & & \\
\end{tabular}

\section{Discussion}

This study is the largest to our knowledge demonstrating that a multidisciplinary tracheostomy service can improve the rate of tracheostomy decannulation and tolerance of oral diet. Others have demonstrated improved time to decannulation only. Speed et al ${ }^{18}$ performed a metaanalysis demonstrating a reduction of total in situ tracheostomy time. This study included 7 articles, all scoring low on internal validity and 2 scoring low for external validity. All studies were inadequately powered, yet 2 studies still reported statistically significant results. The largest study from that analysis by Tobin et $\mathrm{al}^{24}$ noticed an increase in the rate of decannulation over the study period but could only speculate that this may be due to "a more proactive approach to decannulation." Two small studies not included in the meta-analysis demonstrated similar improvements in reducing time to decannulation following implementation of a multidisciplinary tracheostomy team but again failed to report an improvement in the percentage of subjects decannulated. ${ }^{25,26}$

Suboptimal post-ICU tracheostomy care has been implicated and underscored by Mondup et $\mathrm{al}^{7}$ in a Danish survey highlighting inadequate post-ICU tracheostomy management; with only $9 \%$ reported daily follow-up, none reported having decannulation guidelines, and only $10 \%$ delivered education to floor nurses.7,27 A similar survey of 228 ICUs in the United Kingdom found that only $32 \%$ had written protocols for routine tracheostomy follow-up outside the ICU. ${ }^{28}$ These results mirrored similar problems faced by our institution with substantial improvement being made simply by implementing a tracheostomy team. Additionally, our study addresses in detail a majority of the concerns of post-ICU tracheostomy care as described by Mondup. ${ }^{7}$ These details include a defined service line, standardized post-tracheostomy care, a dedicated surgical intensivist, and monthly meetings to unify all the caregivers, provide a platform to discuss new issues, review and 
streamline necessary equipment, and create new standards of care, such as the postoperative care bundle.

Although many bundles or guidelines are adopted into clinical practice, adherence can often be poor. ${ }^{29,30}$ An essential factor in the implementation of our post-tracheostomy care bundle was the empowerment of a small subset of respiratory and speech therapists with physician oversight to track, follow, and provide care for all hospitalized patients with tracheostomy. Barriers to progress were easily identified, and the intensivist-supported rounding process enabled the team to address each concern individually. Likewise, the interdisciplinary approach to protocol development and interprofessional communication, focusing on identification and correction of impediments to progress, appeared to be key. ${ }^{31}$ Of note, the tracheostomy service and post-tracheostomy care bundle process required no additional funding and minimal time obligation. Providers in respiratory therapy and speech language pathology were carefully selected for their motivation and commitment to the goals of the project and were vital to the success of the program.

Oral intake significantly improved following implementation of the tracheostomy service yet continued to improve after the post-tracheostomy care bundle when compared with both baseline and the effects of the tracheostomy service alone. Although not considered in our study, an earlier tracheostomy change may be an important factor in the initiation of oral intake. Fisher et $\mathrm{al}^{32}$ suggest that early tracheostomy change before day 7 is associated with earlier oral intake and speaking valve use. Although Fisher's groups appeared to be equally matched, criteria to meet early or late tracheostomy changes were not disclosed. Also, a potential selection bias was that only 38 of 130 subjects underwent early tracheostomy change. Nonetheless, this study suggests that certain patients could tolerate diet advancement more rapidly. In our protocol, tracheostomy changes occurred as needed based on the speech language pathologist recommendations and according to our tracheostomy decannulation and change guidelines. Thus, a critical step involved our speech language pathologist early in the post-tracheostomy period and considered all subjects for advancement and challenged those who met the criteria for diet regardless of tracheostomy days or tracheostomy change status. Counterintuitively, early involvement of speech language pathologist may be extremely valuable to this cohort. Freeman-Sanderson et $\mathrm{al}^{33}$ retrospectively audited 140 subjects with tracheostomy and found that speech language pathologist consults occurred on average $14 \mathrm{~d}$ following tracheostomy, and oral diet resumed an average of $15 \mathrm{~d}$ after tracheostomy, which may suggest a delay in treatment and underutilization of speech language pathologist resources. In our study, tolerance of oral diet improved as the number of speech consultations increased and as more subjects received early (within 2 d) speech language pathologist consultations.

There were significant differences in APACHE and SOFA scores between all groups. The APACHE scores were performed on admission to the ICU, with SOFA scores calculated at the time of the tracheostomy consult. Both scores were highest in the baseline group, significantly lower in the tracheostomy service group, and then significantly higher again in the post-tracheostomy care bundle group. This could have affected our results because the post-tracheostomy care bundle group had significantly lower severity of illness scores on admission and at time of tracheostomy. In addition, ventilator stay was not significantly shorter in the post-tracheostomy care bundle group compared with the tracheostomy service alone group. This difference may be attributed to a stronger subject on admission and at tracheostomy. The logistic regression analysis was not able to show any correlation with severity of illness or ventilator stay in predicting decannulation. This emphasizes that the design of the post-tracheostomy care bundle was successful in improving quality of care after tracheostomy.

Our study had multiple limitations. First, direct quality of life measures were not collected. The literature does report a reduced quality of life following tracheostomy; one can only infer that removing the tracheostomy tube and improving oral intake would improve quality of life. Other factors important for future investigation, although not analyzed in our study, include time to best diet and cost of care. The potential for cost reduction or return on investment may be realized through decreased tracheostomy-related appliances (tracheostomy tubes, inner cannulas, suction catheters, dressings, tracheostomy ties, oxygen), potential for earlier discharge, reduction in tube feeding materials, and reduction in additional nursing and respiratory care. In addition, subjects were grouped into broad categories based on consulting services; however, specific indications or diagnoses for tracheostomy were not compared, which could have had an effect on outcomes.

\section{Conclusions}

In summary, postoperative tracheostomy care is often overlooked but can have a significant effect on important needs of the patient. An intensivist-led multidisciplinary tracheostomy service with more attention directed at postoperative care is simple to implement and can significantly improve rates of decannulation and tolerance of oral diet. 


\section{Improving Decannulation and Swallowing Function}

\section{REFERENCES}

1. Cox CE, Carson SS, Holmes GM, Howard A, Carey TS. Increase in tracheostomy for prolonged mechanical ventilation in North Carolina, 1993-2002. Crit Care Med 2004;32(11):2219-2226.

2. Mirski MA, Pandian V, Bhatti N, Haut E, Feller-Kopman D, Morad A, et al. Safety, efficiency, and cost-effectiveness of a multidisciplinary percutaneous tracheostomy program. Crit Care Med 2012; 40(6): 1827-1834.

3. Hettige R, Arora A, Ifeacho S, Narula A. Improving tracheostomy management through design, implementation and prospective audit of a care bundle: how we do it. Clin Otolaryngol 2008;33(5):488491.

4. Cetto R. Arora A. Hettige R, Nel M, Benjamin L, Gomez CMH, et al. Improving tracheostomy care: a prospective study of the multidisciplinary approach. Clin Otolaryngol 2011;36(5):482-488.

5. Norwood MG, Spiers P, Bailiss J, Sayers RD. Evaluation of the role of a specialist tracheostomy service: from critical care to outreach and beyond. Postgrad Med J 2004;80(946):478-480.

6. de Mestral C, Iqbal S, Fong N, LeBlanc J, Fata P, Razek T, Khwaja $\mathrm{K}$. Impact of a specialized multidisciplinary tracheostomy team on tracheostomy care in critically ill patients. Can J Surg 2011;54(3): 167-172.

7. Mondrup F, Skjelsager K, Madsen KR. Inadequate follow-up after tracheostomy and intensive care. Dan Med J 2012;59(8):A4481.

8. Ikari T, Sasaki CT. Glottic closure reflex: control mechanisms. Ann Otol Rhinol Laryngol 1980;89(3):220-224.

9. Eibling DE, Gross RD. Subglottic air pressure: a key component of swallowing efficiency. Ann Otol Rhinol Laryngol 1996;105(4):253-258.

10. Gross RD, Mahlmann J, Grayhack JP. Physiologic effects of tracheostomy tube occlusion on the pharyngeal swallow. Ann Otol Rhinol Laryngol 2003;112(2):143-152.

11. Feldman SA, Deal CW, Urquhart W. Disturbance of swallowing after tracheotomy. Lancet 1966;1(7444):954-955.

12. Shaker R, Dodds WJ, Dantas RO, Hogan WJ, Arndorfer RC. Coordination of deglutitive glottic closure with oropharyngeal swallowing. Gastroenterology 1990;98(6):1478-1484.

13. Sasaki CT, Suzuki M, Horiuchi M, Kirchner J. The effect of tracheostomy on the laryngeal closure reflex. Laryngoscope 1977;87(9): 1428-1433.

14. Elpern EH, Scott MG, Petro L, Ries MH. Pulmonary aspiration in mechanically ventilated patients with tracheostomies. Chest 1994; 105(2):563-566

15. Ding R, Logemann JA. Swallow physiology in patients with trach cuff inflated or deflated: a retrospective study. Head Neck 2005; 27(9):809-813.

16. Farri A, Accornero A, Burdese C. Social importance of dysphagia: its impact on diagnosis and therapy. Acta Otorhinolaryngol Ital 2007; 27(2):83-86

17. Gilony D, Gilboa D, Blumstein T, Murad H, Talmi YP, Kronenberg J, Wolf M. Effects of tracheostomy on well-being and body-image perceptions. Otolaryngol Head Neck Surg 2005;133(3):366-371.

18. Speed L, Harding K. Tracheostomy teams reduce total tracheostomy time and increase speaking valve use: a systematic review and metaanalysis. J Crit Care 2013;28(2):216.e1-10.
19. Fulbrook P, Mooney S. Care bundles in critical care: a practical approach to evidence-based practice. Nurs Crit Care 2003;8(6):249255.

20. Resar R, Pronovost P, Haraden C, Simmonds T, Rainey T, Nolan T. Using a bundle approach to improve ventilator care processes and reduce ventilator-associated pneumonia. Jt Comm J Qual Patient Saf 2005;31(5):243-248.

21. Curran E, Murdoch H. Aiming to reduce catheter associated urinary tract infections (CAUTI) by adopting a checklist and bundle to achieve sustained system improvements. J Infect Prev 2009;10(2):57-61.

22. Berenholtz SM, Pronovost PJ, Lipsett PA, Hobson D, Earsing K, Farley JE, et al. Eliminating catheter-related bloodstream infections in the intensive care unit. Crit Care Med 2004;32(10):2014-2020.

23. Dellinger RP, Levy MM, Rhodes A, Annane D, Gerlach H, Opal SM, et al. Surviving sepsis campaign: international guidelines for management of severe sepsis and septic shock: 2012. Crit Care Med 2013;41(2):580-637.

24. Tobin AE, Santamaria JD. An intensivist-led tracheostomy review team is associated with shorter decannulation time and length of stay: a prospective cohort study. Crit Care 2008;12(2):R48.

25. Arora A, Hettige R, Ifeacho S, Narula A. Driving standards in tracheostomy care: a preliminary communication of the St Mary's ENTled multi-disciplinary team approach. Clin Otolaryngol 2008;33(6): 596-599.

26. Cameron TS, McKinstry A, Burt SK, Howard ME, Bellomo R, Brown DJ, et al. Outcomes of patients with spinal cord injury before and after introduction of an interdisciplinary tracheostomy team. Crit Care Resusc 2009;11(1):14-19.

27. Paul F. Tracheostomy care and management in general wards and community settings: literature review. Nurs Crit Care 2010;15(2): 76-85.

28. Krishnan K, Elliot SC, Mallick A. The current practice of tracheostomy in the United Kingdom: a postal survey. Anaesthesia 2005; 60(4):360-364.

29. Brunkhorst FM, Engel C, Ragaller M, Welte T, Rossaint R, Gerlach $\mathrm{H}$, et al. Practice and perception: a nationwide survey of therapy habits in sepsis. Crit Care Med 2008;36(10):2719-2725.

30. Leone M, Ragonnet B, Alonso S, Allaouchiche B, Constantin JM, Jaber $\mathrm{S}$, et al. Variable compliance with clinical practice guidelines identified in a 1-day audit of 66 French adult intensive care units. Crit Care Med 2012;40(12):3189-3195.

31. Gurses AP, Marsteller JA, Ozok AA, Xiao Y, Owens S, Pronovost PJ. Using an interdisciplinary approach to identify factors that affect clinicians' compliance with evidence-based guidelines. Crit Care Med 2010;38(8 Suppl):S282-S291.

32. Fisher DF, Kondili D, Williams J, Hess DR, Bittner EA, Schmidt UH. Tracheostomy tube change before day 7 is associated with earlier use of speaking valve and earlier oral intake. Respir Care 2013;58(2):257-263.

33. Freeman-Sanderson A, Togher L, Phipps P, Elkins M. A clinical audit of the management of patients with a tracheostomy in an Australian tertiary hospital intensive care unit: focus on speech-language pathology. Int J Speech Lang Pathol 2011;13(6):518-525.

This article is approved for Continuing Respiratory Care Education credit. For information and to obtain your CRCE

(free to AARC members) visit www.rcjournal.com

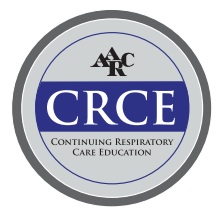

